\title{
Generalized Fractional-Order Discrete-Time Integrator
}

\author{
Dorota Mozyrska $^{1}$ and Piotr Ostalczyk ${ }^{2}$ \\ ${ }^{1}$ Faculty of Computer Science, Bialystok University of Technology, Białystok, Poland \\ ${ }^{2}$ Institute of Applied Computer Science, Lodz University of Technology, tódź, Poland \\ Correspondence should be addressed to Dorota Mozyrska; d.mozyrska@pb.edu.pl
}

Received 10 February 2017; Accepted 23 April 2017; Published 6 July 2017

Academic Editor: Ahmad T. Azar

Copyright (c) 2017 Dorota Mozyrska and Piotr Ostalczyk. This is an open access article distributed under the Creative Commons Attribution License, which permits unrestricted use, distribution, and reproduction in any medium, provided the original work is properly cited.

\begin{abstract}
We investigate a generalization of discrete-time integrator. Proposed linear discrete-time integrator is characterised by the variable, fractional order of integration/summation. Graphical illustrations of an analysis of particular vector matrices are presented. In numerical examples, we show relations between the order functions and element responses.
\end{abstract}

\section{Introduction}

In order to build a dynamic system, one should define specifications to be met, apply synthesis techniques, if available, analyse a mathematical model of a system, and simulate the model on a computer to test the effect of various inputs on the behavior of the resulting system. New classes and categories of systems that could be used as new models are still needed. One of the most important tools is an element called "integrator." In measurements and control applications, an integrator is an element whose output signal is the time integral (in continuous case) or summation (in discrete case) of its input signal. It accumulates the input quantity over a defined time to produce a representative output. For the classical theory, see, for instance, $[1,2]$. An integrator may be treated as a fundamental and is commonly used in constructions of more complicated systems via Kelvin's scheme [2]. This leads to a variety of structures known as realizations. As crucial realizations, those, which reveal such important dynamic properties as stability, controllability, and so forth, are considered. The first-order differential equations can be generalized to the fractional-order ones [3-10]. Hence, we get the fractional-order integrator, which can be used in a modelling of fractional-order dynamic systems.

For discrete-time systems, an equivalent element is called a summator or discrete integrator. This dynamic element is described by linear time-invariant first-order difference equation; see [11, 12]. As a generalization of the classical discrete integrator, we can consider discrete summation of fractional order [3, 13-20]. In this paper, we propose a generalization of the fractional-order discrete integrator and call it the variable-, fractional-order discrete-time integrator.

Besides applications of integrators in mentioned realizations, another important use is the integration action in the PID controllers; see [1,21]. The integration action preserves a zero steady state in the closed-loop systems with typical plants. Different types of the variable-, fractionalorder elements have been proposed in [22, 23]. For constant orders (fractional or integer orders), all integrators are identical. This property is not valid in the variable-, fractionalorder integrators in the mentioned types. Comparing with the model of integrator described in [22], we state here a different and more general model with better motivated initial conditions. Moreover, our investigations of values of coefficients of matrices, that are used for calculations of models, are much more advanced.

The proposed integrator may be used in the variable-, fractional-order digital filters [11, 12], described by related variable-, fractional-order difference equations. In the paper, an equivalent but very useful vector matrix description of the variable-, fractional-order integrator is applied. It becomes a great tool in the variable-, fractional-order integrators description. One should mention that to variable-, fractionalorder difference equations we cannot apply the $Z$-transform.

The paper is organised as follows. After an introduction to the variable-, discrete-, fractional-order calculus, a description of the variable-, fractional-order discrete integrator is 
given in Section 3. The formula for the variable-, fractionalorder integrator response is derived. Our investigations are illustrated by numerical examples.

\section{Preliminaries}

The most important in the evaluation of the variable-, fractional-order backward difference/sum is the kernel function, named after its action the oblivion function. For $k, l \in \mathbb{Z}$ and a given order function $\nu(\cdot): \mathbb{Z} \rightarrow \mathbb{R}$, the function of two discrete variables is defined by its values: $a^{[v(l)]}(k)$. We assume that order functions have values in the interval $[0,1]$.

Definition 1. For $k, l \in \mathbb{Z}$ and a given order function $\nu(\cdot)$, one defines the oblivion function, as a discrete function of two variables, by its values $a^{[v(l)]}(k)$ given as

$$
\begin{aligned}
& a^{[\nu(l)]}(k) \\
& = \begin{cases}0 & \text { for } k<0 \\
1 & \text { for } k=0 \\
(-1)^{k} \frac{\nu(l)[\nu(l)-1] \cdots[\nu(l)-k+1]}{k !} & \text { for } k>0 .\end{cases}
\end{aligned}
$$

It is easy to observe that for opposite values of order function holds the following:

$$
\begin{aligned}
& a^{[-\nu(l)]}(k) \\
& \quad= \begin{cases}0 & \text { for } k<0 \\
1 & \text { for } k=0 \\
\frac{\nu(l)(\nu(l)+1) \cdots(\nu(l)+k-1)}{k !} & \text { for } k>0 .\end{cases}
\end{aligned}
$$

Formula (1) in Definition 1 is equivalent to the following recurrence with respect to $k \in \mathbb{N}$ :

$$
\begin{aligned}
& a^{[\nu(l)]}(0)=1, \\
& a^{[\nu(l)]}(k)=a^{[\nu(l)]}(k-1)\left[1-\frac{\nu(l)+1}{k}\right] \text { for } k \geqslant 1 .
\end{aligned}
$$

In [24], we have proved the following properties for positive values of order function.

Proposition 2 (see [24]). Let the order function have values $0<v(l)<1$ for $l \in \mathbb{Z}$. Then, the following properties hold:

(a) For all $l \in \mathbb{Z}$ and $k \in \mathbb{N}_{1}, a^{[v(l)]}(k)<0$.

(b) For all $l \in \mathbb{Z}$, the sequence $\left(a^{[v(l)]}(k)\right)_{k \in \mathbb{N}_{1}}$ is increasing.

(c) For each increasing and bounded order function $\nu(\cdot)$ with values in $(0,1)$ and for each $l \in \mathbb{N}$, there is $m_{l}$ such that for $k \geq m_{l}$ the sequence $\left(a^{[\nu(l)]}(k)\right)_{l \in \mathbb{N}}$ is increasing; that is, for $k \geq m_{l}$,

$$
a^{[v(l+1)]}(k)>a^{[v(l)]}(k) .
$$

Particularly for order functions with values in $1 / 2 \leq$ $v(l)<1$, the border $m_{l}=2$.

(d) For each increasing and bounded order function $v(\cdot)$ with values in $(0,1)$, there is $m_{k} \in \mathbb{N}_{1}$ such that for $k \geq m_{k}$

$$
a^{[\nu(k+1)]}(k+1)>a^{[\nu(k)]}(k) .
$$

In the sequel, we need to prove parallel properties for oblivion function with negative values of order function with values $-v(l) \in(-1,0)$ for $l \in \mathbb{Z}$.

Proposition 3. Let one assume that, $\forall l \in \mathbb{Z}, v(l) \in(0,1)$. Then the following properties are satisfied:

(a) For all $l \in \mathbb{Z}$ and $k \in \mathbb{N}_{1}, a^{[-\nu(l)]}(k)>0$.

(b) For all $l \in \mathbb{Z}$, the sequence $\left(a^{[-\nu(l)]}(k)\right)_{k \in \mathbb{N}_{1}}$ is decreasing.

(c) For decreasing and bounded order function $v(\cdot)$ with values in $(0,1)$ and for each $l \in \mathbb{N}$, the sequence $\left(a^{[-v(l)]}(k)\right)_{l \in \mathbb{N}}$ is decreasing; that is, for $k \geq \mathbb{N}_{1}$,

$$
a^{[-\nu(l+1)]}(k)<a^{[-\gamma(l)]}(k) .
$$

(d) For decreasing and bounded order function $\nu(\cdot)$ with values in $(0,1)$ and for each $l \in \mathbb{N}$ holds

$$
a^{[-\nu(k+1)]}(k+1)<a^{[-\nu(k)]}(k) .
$$

Proof. For $l \in \mathbb{Z}$, we have that $a^{[-\nu(l)]}(0)=1$ and for $k>0$

$$
a^{[-\nu(l)]}(k)=\frac{\nu(l)(\nu(l)+1) \cdots(\nu(l)+k-1)}{k !} .
$$

Then, we directly have points (a), (b), and (c). In (b), we need additionally to notice that $(\nu(l)-1) / k<0$, which gives $a^{[-\gamma(l)]}(k+1)<a^{[-\gamma(l)]}(k)$. We do the next calculations to receive what we claim in point $(\mathrm{d})$. We have the following:

$$
\begin{aligned}
& a^{[-\nu(k+1)]}(k+1)-a^{[-\nu(k)]}(k) \\
& \quad=\left(1-\frac{-v(k+1)+1}{k+1}\right) a^{[-\nu(k+1)]}(k)-a^{-[\nu(k)]}(k) .
\end{aligned}
$$

Moreover, as $0<v(k+1)<1$, then $0<1+(\nu(k+1)-1) /(k+$ $1)<1$ for $k \leq 1$. Knowing that $a^{[-\nu(k+1)]}(k)>0$, we receive $(1-(-v(k+1)+1) /(k+1)) a^{[-\nu(k+1)]}(k)<a^{[-\nu(k+1)]}(k)$. Hence,

$$
\begin{gathered}
a^{[-\nu(k+1)]}(k+1)-a^{[-\nu(k)]}(k) \\
<a^{-[\nu(k+1)]}(k)-a^{[v(k)]}(k)
\end{gathered}
$$

and then from point (c) we have the thesis. 
In the next definition, the Grünwald-Letnikov fractionalorder backward difference (GL-FOBD) is generalized to the Grünwald-Letnikov variable-, fractional-order backward difference (GL-VFOBD) in part (a) and to the Grünwald-Letnikov variable-, fractional-order backward difference with initialization (GL-VFOBDwI) in part (b). For definition and properties of the Grünwald-Letnikov fractional-order backward difference (GL-FOBD) for constant order, we refer to $[10,18,25,26]$.

Definition 4. Let $f$ be a discrete-variable bounded real valued function.

(a) The Grünwald-Letnikov variable-, fractionalorder backward difference with initialization (GLVFOBDwI) with an order function $v: \mathbb{Z} \rightarrow \mathbb{R}_{+} \cup\{0\}$ is defined as an infinite sum, provided that the series is convergent:

$$
\begin{aligned}
& \left({ }_{-\infty}^{\mathrm{GL}} \Delta^{[\nu(k)]} f\right)(k)=\sum_{i=0}^{\infty} a^{[\nu(k)]}(i) f(k-i)
\end{aligned}
$$

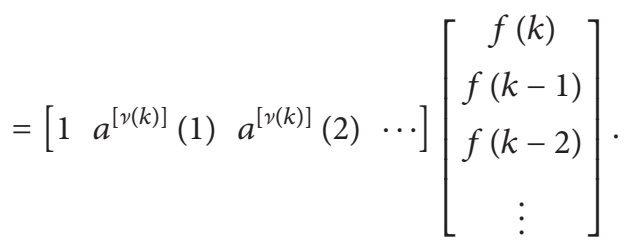

(b) The Grünwald-Letnikov variable-, fractional-order backward difference (GL-VFOBD) with an order function $v: \mathbb{Z} \rightarrow \mathbb{R}_{+} \cup\{0\}$ started at $k_{0}$ is defined as a finite sum

$$
\begin{aligned}
& \left({ }_{k_{0}}^{G L} \Delta^{[\nu(k)]} f\right)(k)=\sum_{i=k_{0}}^{k} a^{[\nu(k)]}\left(i-k_{0}\right) f\left(k+k_{0}-i\right) \\
& =\sum_{i=0}^{k-k_{0}} a^{[\nu(k)]}(i) f(k-i)
\end{aligned}
$$

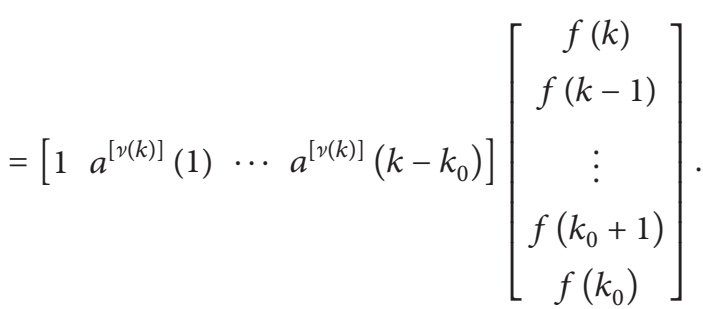

For $k_{0}=0$, the GL-VFOBD becomes a discrete convolution: $\left({ }_{0}^{G L} \Delta^{[\gamma(k)]} f\right)(k)=(\mathfrak{a} * f)(k)=(f * \mathfrak{a})(k)$, where $\mathfrak{a}(k):=$ $a^{[v(k)]}(k)$. In particular case of constant order function, we have the following (for $k>k_{0}$ and $k_{0}$ can be finite or $k_{0}=$ $-\infty)$ :

(i) $\left.{ }_{k_{0}}^{G L} \Delta^{[0]} f\right)(k)=f(k)$;

(ii) $\left.{ }_{k_{0}}^{G L} \Delta^{[1]} f\right)(k)=f(k)-f(k-1)$; (iii) $\left({ }_{k_{0}}^{G L} \Delta^{[-1]} f\right)(k)=\sum_{i=0}^{k-k_{0}} f(k-i)$, if only the summation exists.

Next, one assumes that $v(k)=0$ for $k \leqslant k_{0}-1$. Equality (11) is defined for any $k \geqslant k_{0}>-\infty$, so it is also valid for any $k-1, k-2, \ldots, k_{0}+1, k_{0}, k_{0}-1, \ldots$.

Collecting all such equalities like (11) in one vector matrix form, one obtains

$$
\left({ }_{-\infty}^{G L} \Delta^{[\gamma(k)]} \mathbf{f}\right)(k)={ }_{-\infty} \mathbf{A}_{k_{0}, k}^{[\gamma(k)]}\left[\begin{array}{l}
\mathbf{f}(k) \\
\mathbf{f}_{k_{0}-1}
\end{array}\right],
$$

where

$$
\begin{aligned}
& \mathbf{f}(k)=\left[\begin{array}{c}
f(k) \\
f(k-1) \\
\vdots \\
f\left(k_{0}+1\right) \\
f\left(k_{0}\right)
\end{array}\right], \\
& \mathbf{f}_{k_{0}-1}=\left[\begin{array}{c}
f\left(k_{0}-1\right) \\
f\left(k_{0}-2\right) \\
\vdots
\end{array}\right] \text {, } \\
& \left({ }_{-\infty}^{G L} \Delta^{[\nu(k)]} \mathbf{f}\right)(k)=\left[\begin{array}{c}
{ }_{-\infty}^{G L} \Delta^{[\nu(k)]} f(k) \\
\vdots \\
{ }_{-\infty} \Delta^{\left[\nu\left(k_{0}\right)\right]} f\left(k_{0}\right)
\end{array}\right] \text {, } \\
& { }_{-\infty} \mathbf{A}_{k_{0}, k}^{[\gamma(k)]}:=\left[\begin{array}{lll}
{ }_{k_{0}} & \mathbf{A}_{k}^{[\gamma(k)]} & -\infty \\
{ }_{-\infty} \mathbf{I}_{k_{0}-1}^{[\gamma(k)]}
\end{array}\right] .
\end{aligned}
$$

Inside matrix (15) we extract the following parts:

$$
k_{0} \mathbf{A}_{k}^{[\nu(k)]}:=\left[\begin{array}{cccc}
1 & a^{[\nu(k)]}(1) & \cdots & a^{[\nu(k)]}\left(k-k_{0}\right) \\
0 & 1 & \cdots & a^{[\nu(k-1)]}\left(k-k_{0}-1\right) \\
\vdots & \vdots & & \vdots \\
0 & 0 & \cdots & 1
\end{array}\right],
$$

$$
\begin{aligned}
& { }_{-\infty} \mathbf{I}_{k_{0}-1}^{[\gamma(k)]} \\
& :=\left[\begin{array}{ccc}
a^{[\nu(k)]}\left(k-k_{0}+1\right) & a^{[\nu(k)]}\left(k-k_{0}+2\right) & \ldots \\
a^{[\nu(k-1)]}\left(k-k_{0}\right) & a^{[\nu(k-1)]}\left(k-k_{0}+1\right) & \ldots \\
\vdots & \vdots & \\
a^{[\nu(0)]}(1) & a^{[\nu(0)]}(2) & \ldots
\end{array}\right] .
\end{aligned}
$$


For $v=1$ and $k_{0}=0$, appropriate matrices have the following forms:

$$
\begin{aligned}
k_{0} \mathbf{A}_{k}^{[-1]} & =\left[\begin{array}{cccccc}
1 & 1 & 1 & \cdots & 1 & 1 \\
0 & 1 & 1 & \cdots & 1 & 1 \\
0 & 0 & 1 & \cdots & 1 & 1 \\
\vdots & \vdots & \vdots & & \vdots & \vdots \\
0 & 0 & 0 & \cdots & 1 & 1 \\
0 & 0 & 0 & \cdots & 0 & 1
\end{array}\right], \\
k_{0} \mathbf{A}_{k}^{[1]} & =\left[\begin{array}{cccccc}
1 & -1 & 0 & \cdots & 0 & 0 \\
0 & 1 & -1 & \cdots & 0 & 0 \\
0 & 0 & 1 & \cdots & 0 & 0 \\
\vdots & \vdots & \vdots & & \vdots & \vdots \\
0 & 0 & 0 & \cdots & 1 & -1 \\
0 & 0 & 0 & \cdots & 0 & 1
\end{array}\right] .
\end{aligned}
$$

Moreover, it is worth noticing that ${ }_{k_{0}} \mathbf{A}_{k}^{[0]}=\mathbf{1}_{k-k_{0}+1}$, which is the $\left(k-k_{0}+1\right) \times\left(k-k_{0}+1\right)$-dimensional identity matrix.

We give now the series of properties of finite dimensional matrices ${ }_{k_{0}} \mathbf{A}_{k}^{[\nu(k)]}$ and ${ }_{k_{0}} \mathbf{A}_{k}^{[-\nu(k)]}$ and their inverses, for order function with values $\nu(k) \in(0,1]$.

Proposition 5 (see [24]). Assume that for all $k \geq k_{0}, v(k) \epsilon$ $(0,1]$ and $\nu(k)=0$ for $k \leq k_{0}-1$. Then, all elements of the inverse matrix $\left({ }_{k_{0}} \mathbf{A}_{k}^{[v(k)]}\right)^{-1}$ are nonnegative.

Proposition 6 (see [24]). For an order function $v(k)>0$ and a constant order $\nu_{c}>0$, the following equality holds: ${ }_{0} \mathbf{A}_{k}^{[\nu(k)]}{ }_{0} \mathbf{A}_{k}^{\left(v_{c}\right)}={ }_{0} \mathbf{A}_{k}^{\left[v(k)+v_{c}\right]}$.

By direct calculations, one can check that ${ }_{0} \mathbf{A}_{k}^{\left(\nu_{c}\right)}{ }_{0} \mathbf{A}_{k}^{[v(k)]} \neq$ ${ }_{0} \mathbf{A}_{k}^{\left[\nu(k)+v_{c}\right]}$. Proposition 6 confirms the fact that for $\nu(k)=\nu=$ const, we have ${ }_{0} \mathbf{A}_{k}^{(v)} \mathbf{A}_{k}^{\left(v_{c}\right)}={ }_{0} \mathbf{A}_{k}^{\left(\nu+v_{c}\right)}$. As a consequence of the last equality, one has the following ${ }_{k_{0}} \mathbf{A}_{k}^{(v)}=\left({ }_{k_{0}} \mathbf{A}_{k}^{(-v)}\right)^{-1}$. By direct calculations, we can check that

$$
\left({ }_{k_{0}} \mathbf{A}_{k}^{[\nu(k)]}\right)^{-1} \neq\left({ }_{k_{0}} \mathbf{A}_{k}^{[-\gamma(k)]}\right) .
$$

The crucial matrix in the VFODI response is ${ }_{k_{0}} \mathbf{A}_{k}^{[-v(k)]}$. We prove the following in [22].

Proposition 7 (see [22]). For order functions with values $0 \leq$ $\nu(k) \leqslant 1$ for $k \geq k_{0}$, all elements of the matrix ${ }_{k_{0}} \mathbf{A}_{k}^{[-\nu(k)]}$ are nonnegative.

Proposition 8 (see [22]). For two order functions with values $0<v_{1}(k)<v_{2}(k) \leqslant 1$, for $k \geq k_{0}$, all elements of the matrix $\left(a_{i j}\right)={ }_{k_{0}} \mathbf{A}_{k}^{\left[-v_{1}(k)\right]}$ are less than or equal to these of the matrix $\left(b_{i j}\right)={ }_{k_{0}} \mathbf{A}_{k}^{\left[-v_{2}(k)\right]}$; that is, $a_{i j} \leq b_{i j}$.

\section{Variable-, Fractional-Order Difference Integrator (VFODI)}

3.1. Description of the VFODI. Let $u$ be the given function $u$ : $\mathbb{Z} \mapsto \mathbb{R}$ and $f: \mathbb{R}^{2} \mapsto \mathbb{R}$ be given bounded function of two variables. Let $\bar{f}(k):=f(y(k-1), u(k)), k \in \mathbb{Z}$. The variable-, fractional-order discrete-time difference equation for the pair of orders $(\nu, \mu)$ is described by the following fractional-order difference equation:

$$
\left({ }_{-\infty}^{G L} \Delta^{[v(k)]} y\right)(k)=\left({ }_{k_{0}}^{G L} \Delta^{[-\mu(k)]} \bar{f}\right)(k), \quad k \geq k_{0}
$$

with initial conditions

$$
\mathbf{y}_{k_{0}-1}=\left[\begin{array}{c}
y\left(k_{0}-1\right) \\
y\left(k_{0}-2\right) \\
\vdots
\end{array}\right]
$$

and values $u(k)=0$ for $k<k_{0}$.

From definitions of fractional operators, (21) has the following recurrence solution:

$$
\begin{aligned}
y(k)= & -\sum_{i=1}^{\infty} a^{[v(k)]}(i) y(k-i) \\
& +\sum_{i=0}^{k-k_{0}} a^{[-\mu(k)]}(i) f(y(k-1-i), u(k-i)), \\
& k \geq k_{0} .
\end{aligned}
$$

In the simplest situations, we present solutions given by (23) in the form

(i) for $\nu \equiv 0, \mu \equiv 0$,

$y(k)=f(y(k-1), u(k))$ and this looks like classical difference equation;

(ii) for $\nu \equiv 1, \mu \equiv 0$,

$y(k)=y(k-1)+f(y(k-1), u(k))$ and this is the case of the so-called nabla operator equation;

(iii) for $\nu \equiv 0, \mu \equiv 1$,

$$
y(k)=\sum_{i=1}^{k-k_{0}} f(y(k-1-i), u(k-i)) ;
$$

(iv) for $\nu \equiv 1, \mu \equiv 1$,

$$
y(k)=y(k-1)+\sum_{i=1}^{k-k_{0}} f(y(k-1-i), u(k-i)) .
$$


In the next definition of variable-, fractional-order difference integrator (VFODI), we assume that $f(y(k-1), u(k))=$ $b_{0} u(k)$. Let us introduce the following notation:

$$
\begin{aligned}
& \mathbf{y}(k)=\left[\begin{array}{c}
y(k) \\
y(k-1) \\
\vdots \\
y\left(k_{0}+1\right) \\
y\left(k_{0}\right)
\end{array}\right], \\
& \mathbf{u}(k)=\left[\begin{array}{c}
u(k) \\
u(k-1) \\
\vdots \\
u\left(k_{0}+1\right) \\
u\left(k_{0}\right)
\end{array}\right], \\
& y(k)=\left[\begin{array}{c}
y(k) \\
y(k-1) \\
\vdots
\end{array}\right], \quad \text { for } k \in \mathbb{Z} .
\end{aligned}
$$

If $k \geq k_{0}$, then we can write $Y(k)$ in the following way $Y(k)=$ $\left[\begin{array}{c}\mathbf{y}(k) \\ \mathbf{y}_{k_{0}-1}\end{array}\right]$.

The variable-, fractional-order difference integrator (VFODI) is described by the following fractional-order difference matrix-vector equation:

$$
\left({ }_{-\infty} \mathbf{A}_{k}^{[\nu(k)]}\right) \cdot Y(k)=b_{0}\left({ }_{k_{0}} \mathbf{A}_{k}^{[-\mu(k)]}\right) \mathbf{u}(k)
$$

with initial condition $Y\left(k_{0}-1\right)=\mathbf{y}_{k_{0}-1}$. On the right side of (25), we use finite matrix ${ }_{k_{0}} \mathbf{A}_{k}^{[-\mu(k)]}$ as on the left side there is a rectangular (finite rows and infinite columns) matrix operator. It is because we claim as in the classical cases that values $u(k)=0$ for $k<k_{0}$. Then, even if we write infinite operator matrix on the right side, it will give the same action as multiplication by an infinite number of zeros of $u(\cdot)$.

Proposition 9. Equation (25) with initial condition $Y\left(k_{0}-\right.$ $1)=\mathbf{y}_{k_{0}-1}$ has the solution given by the following description:

$$
\begin{aligned}
\mathbf{y}(k)= & \left({ }_{k_{0}} \mathbf{A}_{k}^{[\nu(k)]}\right)^{-1} \\
& \cdot\left(b_{0 k_{0}} \mathbf{A}_{k}^{[-\mu(k)]} \mathbf{u}(k)-{ }_{-\infty} \mathbf{I}_{k_{0}-1}^{[\nu(k)]} \mathbf{y}_{k_{0}-1}\right) .
\end{aligned}
$$

Proof. The proof follows from the fact that taking into account the initial conditions vector $Y\left(k_{0}-1\right)$ and the partition of the matrices ${ }_{-\infty} \mathbf{A}_{k_{0}, k}^{[\gamma(k)]}$, we have from (25)

$$
\left[{ }_{k_{0}} \mathbf{A}_{k}^{[\nu(k)]}{ }_{-\infty} \mathbf{I}_{k_{0}-1}^{[\nu(k)]}\right]\left[\begin{array}{l}
\mathbf{y}(k) \\
\mathbf{y}_{k_{0}-1}
\end{array}\right]=b_{0}\left({ }_{k_{0}} \mathbf{A}_{k}^{[-\mu(k)]}\right) \mathbf{u}(k) .
$$

It is possible as matrices ${ }_{k_{0}} \mathbf{A}_{k}^{[\gamma(k)]}$ and ${ }_{k_{0}} \mathbf{A}_{k}^{[-\mu(k)]}$ are always nonsingular.
For example, for $k=1$ and zero initial conditions vector $Y\left(k_{0}-1\right)=0$ with $k_{0}=0$, we have that

$$
\begin{aligned}
& \mathbf{y}(1)=\left[\begin{array}{l}
y(1) \\
y(0)
\end{array}\right]=b_{0}\left(\mathbf{A}_{1}^{[\nu(1)]}\right)^{-1} \mathbf{A}_{1}^{[-\mu(1)]}\left[\begin{array}{l}
u(1) \\
u(0)
\end{array}\right], \\
& \mathbf{y}(1)=b_{0}\left[\begin{array}{c}
u(1)+u(0)(\nu(1)+\mu(1)) \\
u(0)
\end{array}\right] .
\end{aligned}
$$

Hence, $y(0)=b_{0} u(0)$ and $y(1)=b_{0}(u(1)+u(0)(\nu(1)+\mu(1)))$.

Proposition 10. The VFODI of form (25) with fractional orders satisfying the condition that for each $k \geq k_{0}, \nu(k)+$ $\mu(k)=1$ and zero initial conditions vector $Y\left(k_{0}-1\right)=\mathbf{0}$ is a classical first-order summator (discrete-time integrator).

Proof. By the assumption $-\mu(k)=\nu(k)-1$, then

$$
{ }_{k_{0}} \mathbf{A}_{k}^{[\nu(k)]} \mathbf{y}(k)=b_{0 k_{0}} \mathbf{A}_{k}^{[\nu(k)-1]} \mathbf{u}(k) .
$$

Based on investigations in [24], we know that (29) is equivalent to

$$
{ }_{k_{0}} \mathbf{A}_{k}^{[\nu(k)]} \mathbf{y}(k)=b_{0 k_{0}} \mathbf{A}_{k}^{[\nu(k)]}{ }_{k_{0}} \mathbf{A}_{k}^{[-1]} \mathbf{u}(k) .
$$

Matrix ${ }_{k_{0}} \mathbf{A}_{k}^{[\gamma(k)]}$ is always nonsingular as an upper triangular matrix with ones on the main diagonal. Hence, from (30) one immediately gets $\mathbf{y}(k)=b_{0 k_{0}} \mathbf{A}_{k}^{[-1]} \mathbf{u}(k)$ and equivalently

$$
k_{0} \mathbf{A}_{k}^{[1]} \mathbf{y}(k)=b_{0} \mathbf{u}(k) .
$$

Matrix $_{k_{0}} \mathbf{A}_{k}^{(1)}$ has the form (19). From the first row of (31) with (19), one obtains $y(k)-y(k-1)=b_{0} u(k)$ or equivalently $y(k)=b_{0} \sum_{i=0}^{k} u(i)$.

Example 11. In the numerical example, usefulness of the VFODI is presented. In the first one, the discrete unit step responses of the VFODI for assumed fractional-order function are presented. In the second example, some particular application of the VFODI is shown.

Let us consider two fractional-order functions given by their values for $k \in \mathbb{N}$ :

$$
\begin{gathered}
\nu(k)=0.5-0.5 e^{-0.01 k}, \\
\mu(k)=-0.5+0.5 e^{-0.05 k} .
\end{gathered}
$$

In Figure 1, plots of order functions $\nu(k)$, black line, and $\mu(k)$, red line, are presented. As a consequence in Figures 2(a) and 2(b), images and 3D matrix values are presented.

In Figures 3(a) and 3(b), images and 3D matrix values of $\left[{ }_{k_{0}} \mathbf{A}_{k}^{[\gamma(k)]}\right]^{-1}$ are presented, where $\left(p_{1}, p_{2}\right)$ are pixels positions and $c\left(p_{1}, p_{2}\right)$ are the color representations.

The image and values of matrix $\left[{ }_{k_{0}} \mathbf{A}_{k}^{[\nu(k)]}\right]_{k_{0}}^{-1} \mathbf{A}_{k}^{[-\mu(k)]}$ are given in Figures 4(a) and 4(b).

3.2. Particular Forms of VFODI. We consider here two special cases of (25), the first one for $\mu(k) \equiv 0$ and the second one for $\nu(k) \equiv 0$. 


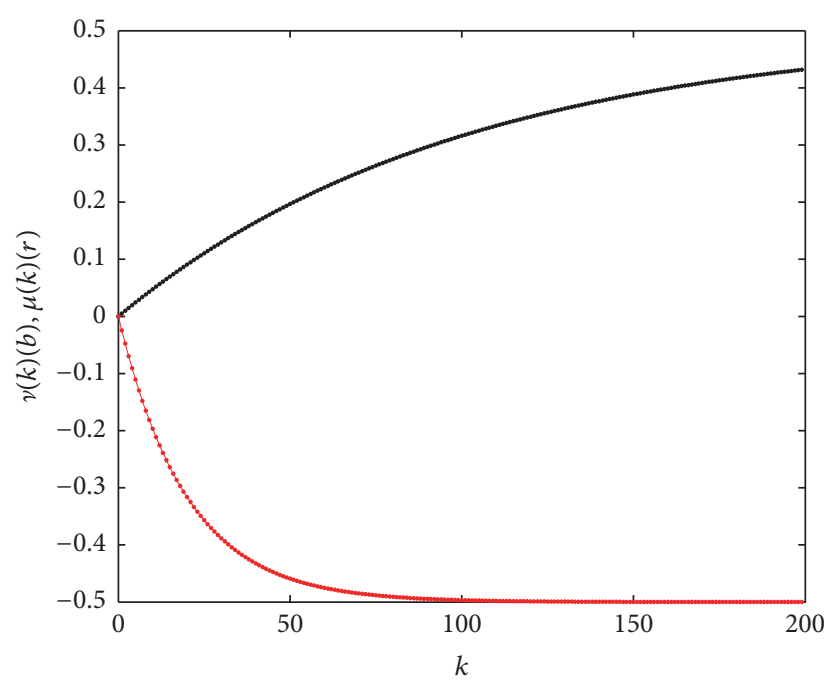

FIgURE 1: Plots of order functions defined by formula (32).

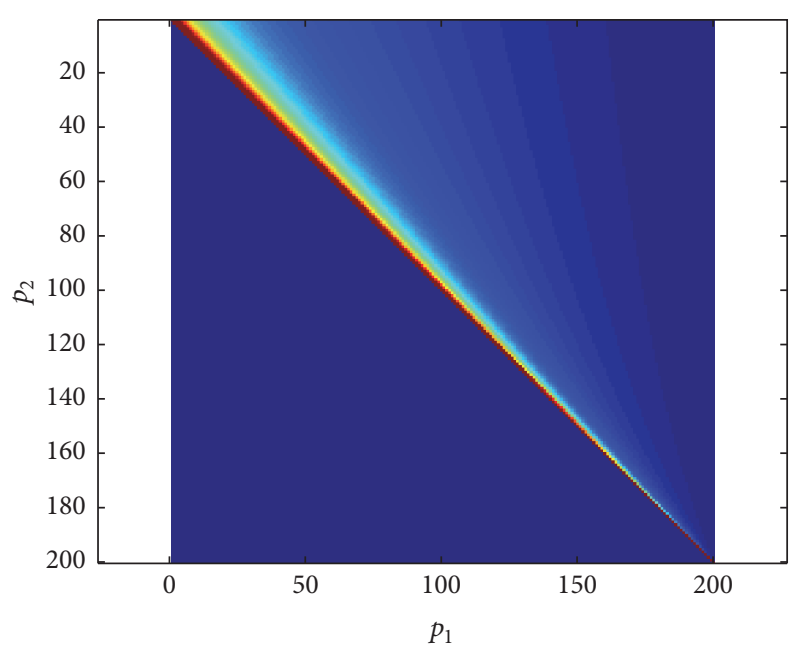

(a) Image of the matrix

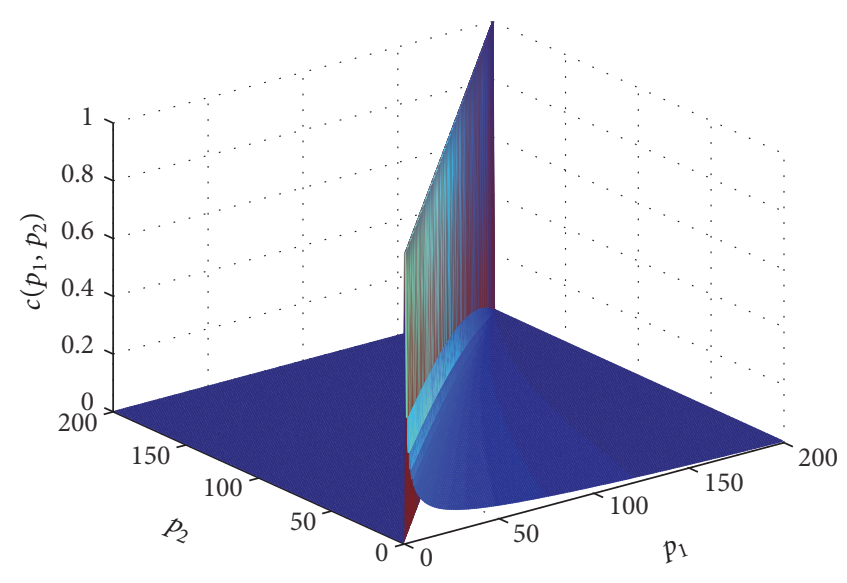

(b) $3 \mathrm{D}$ plot of values of the matrix

FIGURE 2: Image of the matrix and 3D plot of values of the matrix $\left[{ }_{k_{0}} \mathbf{A}_{k}^{[v(k)]}\right]^{-1}$.

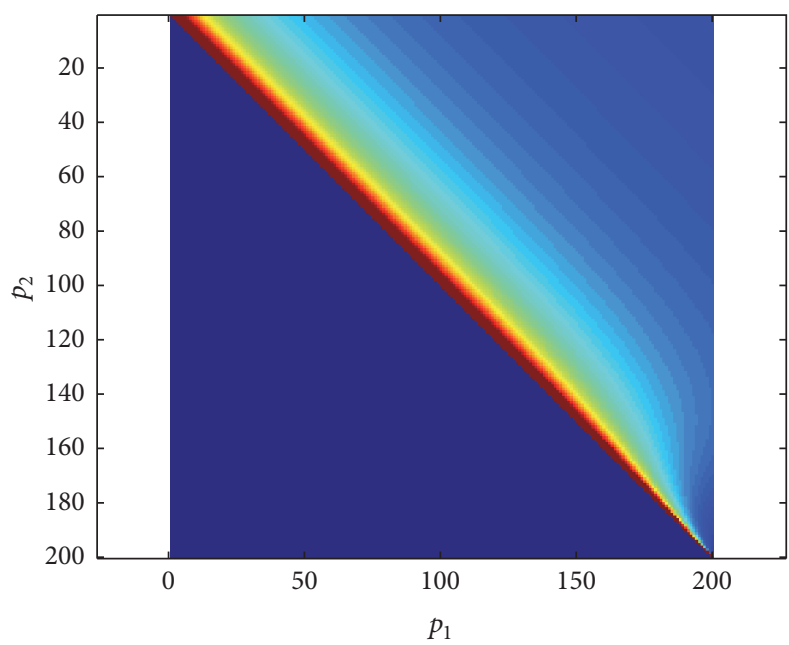

(a) Image of the matrix

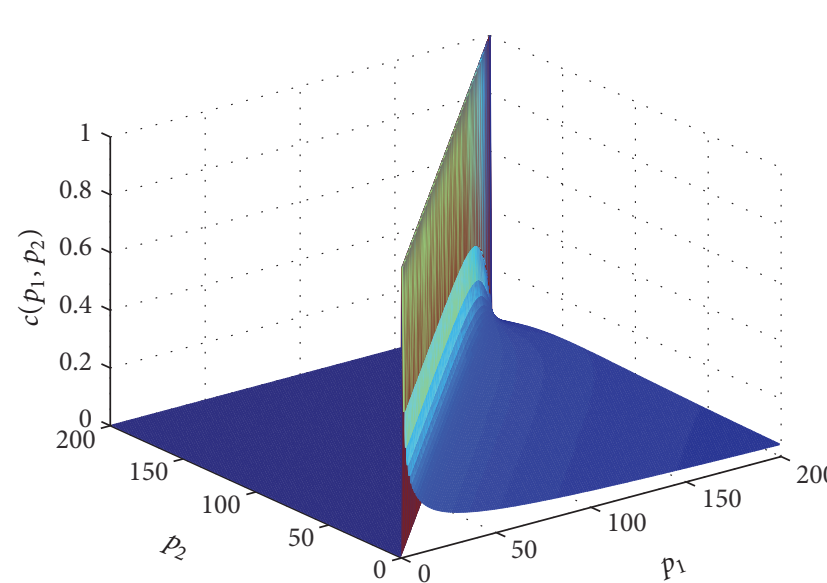

(b) 3D plot of values of the matrix

FIGURE 3: Image of the matrix and 3D plot of values of the matrix $k_{0} \mathbf{A}_{k}^{[-\mu(k)]}$. 


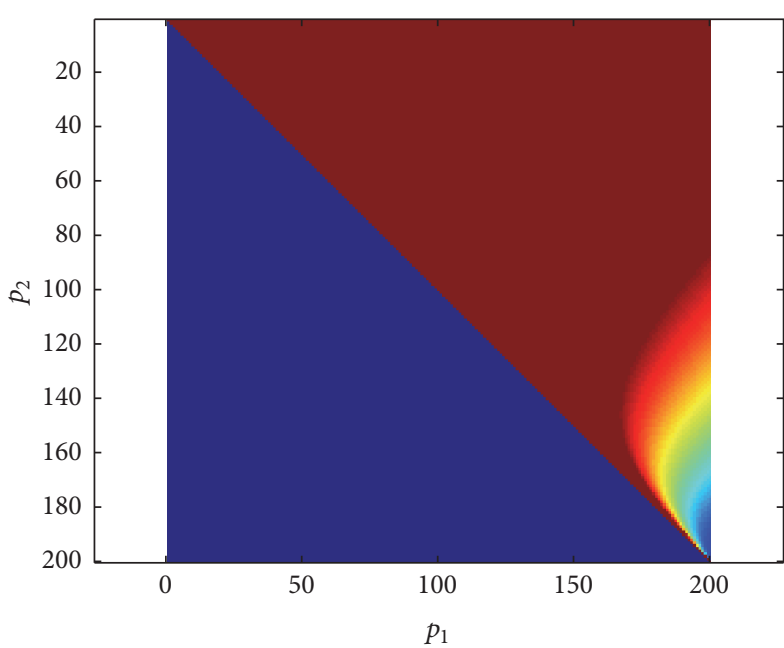

(a) Image of the matrix

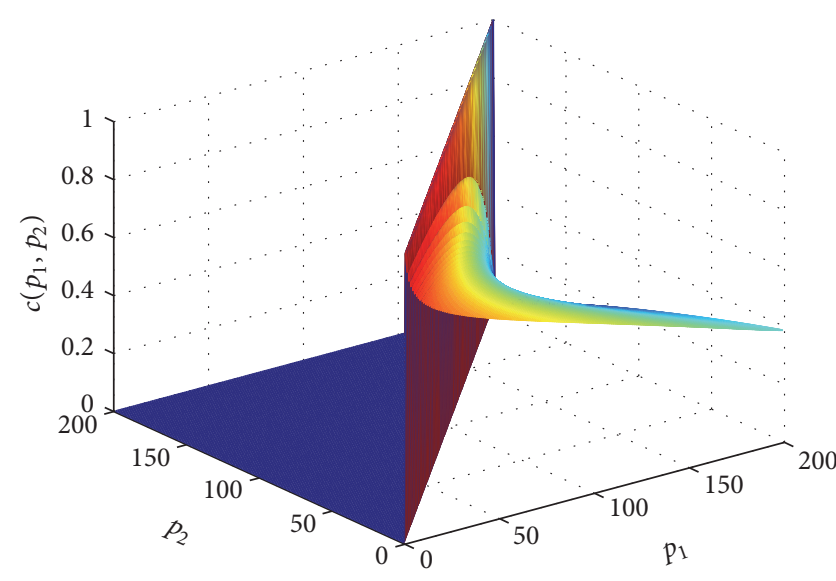

(b) 3D plot of values of the matrix

FIgURE 4: Image of the matrix and 3D plot of values of the matrix $\left[k_{0} \mathbf{A}_{k}^{[v(k)]}\right]_{k_{0}}^{-1} \mathbf{A}_{k}^{[-\mu(k)]}$.

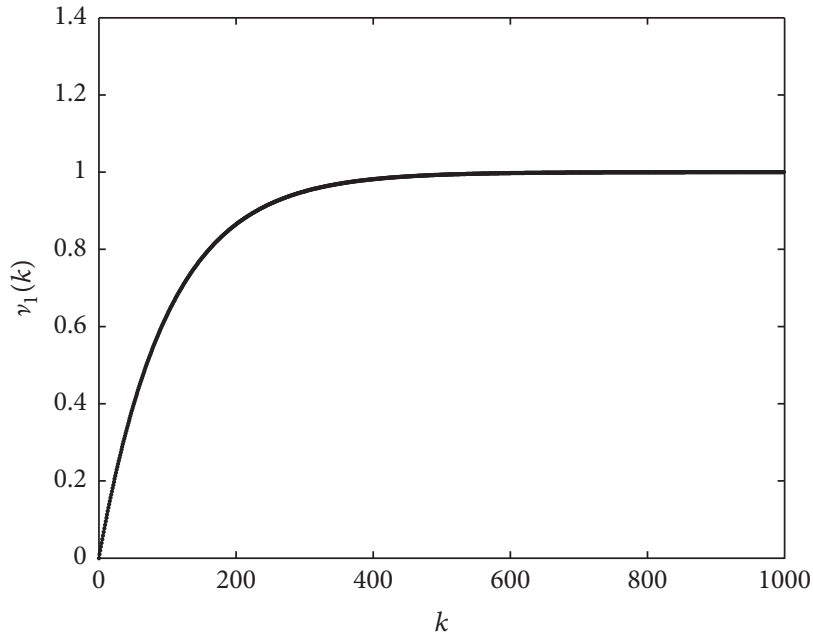

(a) The plot of the order function

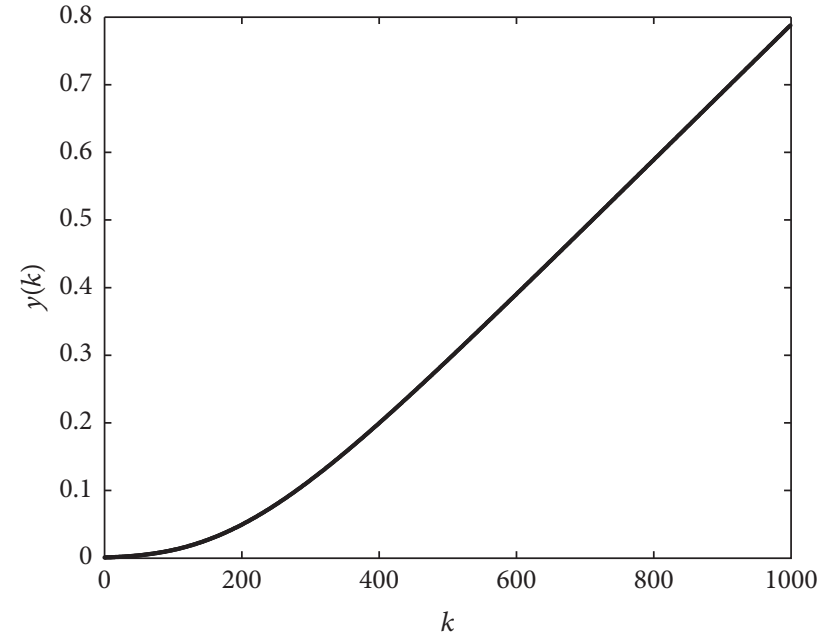

(b) The unit step response

FIGURE 5: Plots of the order function $v_{1}$ given by (35) and the unit step response of the 1st form of VFODI represented by formula (34) with $b_{0}=0.001, k_{0}=0$.

(i) Let us consider $\mu(k) \equiv 0$. Then, (25) takes form

$$
\left({ }_{-\infty} \mathbf{A}_{k}^{[v(k)]}\right) \cdot Y(k)=b_{0} \mathbf{u}(k)
$$

and the VFODI response, with initial condition $Y\left(k_{0}-\right.$ 1) $=\mathbf{y}_{k_{0}-1}$, is described by the formula proved in Proposition 9:

$$
\begin{aligned}
\mathbf{y}(k)= & b_{0}\left({ }_{k_{0}} \mathbf{A}_{k}^{[v(k)]}\right)^{-1} \cdot \mathbf{u}(k)-\left({ }_{k_{0}} \mathbf{A}_{k}^{[\nu(k)]}\right)^{-1} \\
& \cdot{ }_{-\infty} \mathbf{I}_{k_{0}-1}^{[\nu(k)]} \mathbf{y}_{k_{0}-1} .
\end{aligned}
$$

One should emphasise that the initial condition vector is infinite dimensional. This is characteristic for systems with "memory" of the state.
Example 12. In the following numerical example, we examine the discrete unit step responses of the first form of the VFODI. We present plots of order functions and solutions of VFODI, given by (34) with $b_{0}=0.001, k_{0}=0$.

(a) Let us consider order function given by

$$
\nu_{1}(k)=1-e^{-0.01 k} .
$$

The order function $v_{1}$ is plotted in Figure 5(a). The graph of the unit step response is given in Figure 5(b). The considered order function is characterised by two time intervals separated by a time instant $k=$ 400. In the first one, the order function increases monotonically from 0 to 1 . Hence, the summation force is weaker but growing. In the second interval, 


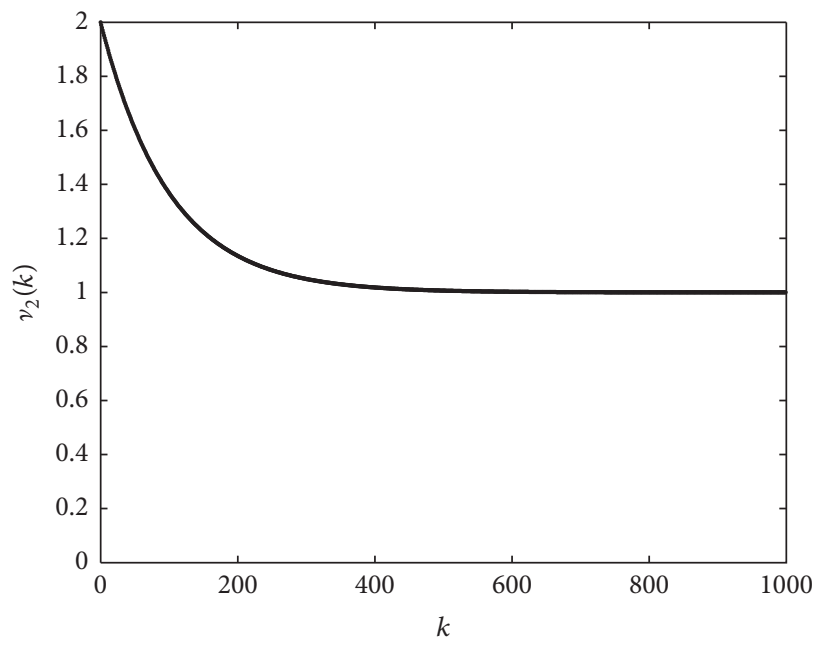

(a) The plot of the order function

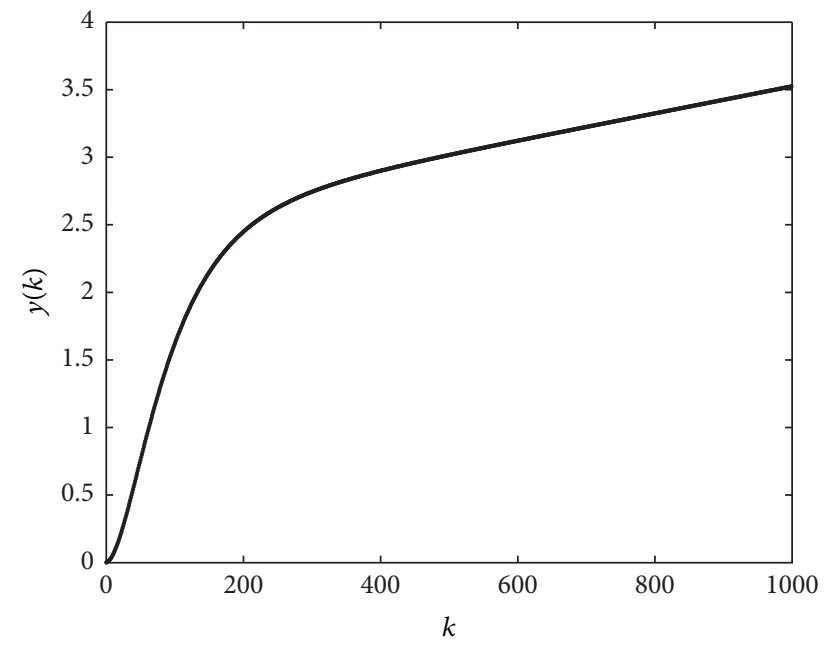

(b) The unit step response

FIGURE 6: Plots of the order function $v_{2}$ given by (36) and the unit step response of the 1st form of VFODI represented by formula (34) with $b_{0}=0.001, k_{0}=0$.

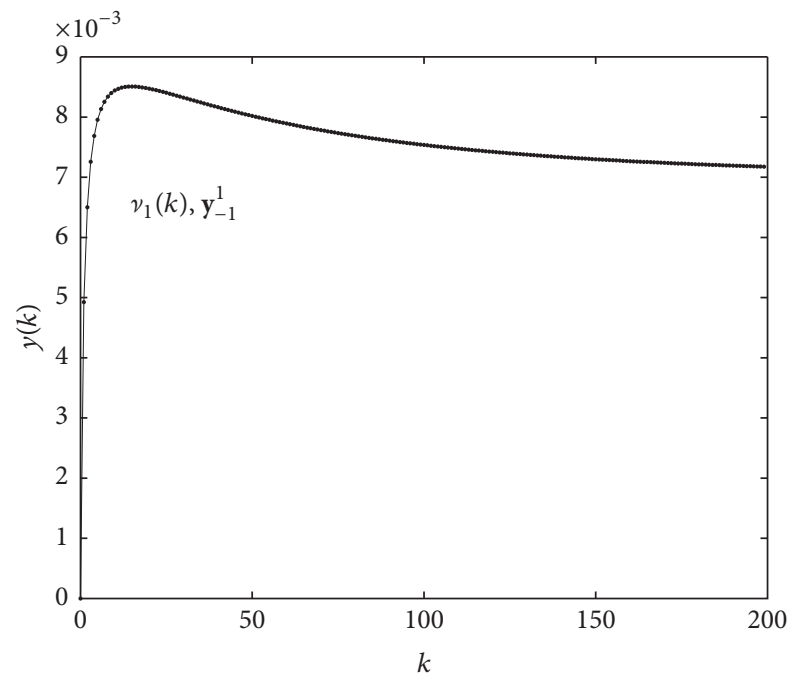

(a) Plot of response to $\mathbf{y}_{-1}^{1}$

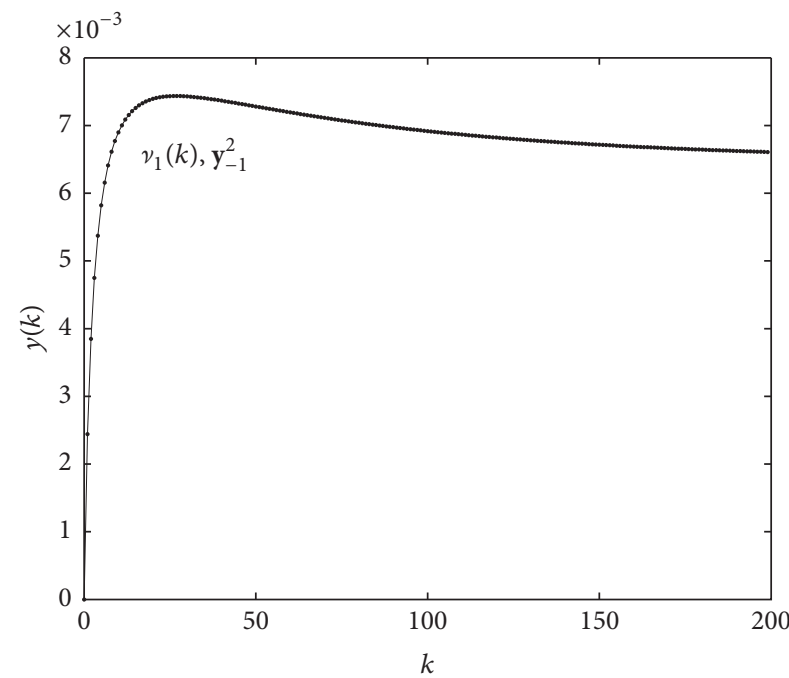

(b) Plot of response to $\mathbf{y}_{-1}^{2}$

FIGURE 7: The VFOI homogenous responses to $\mathbf{y}_{-1}^{1}$ and $\mathbf{y}_{-1}^{2}$ with $b_{0}=0.001, k_{0}=0$ in formula (34) and the order $\nu_{1}(k)=1-e^{-0.01 k}$.

the function is almost constant. This means that the VFODI acts as a classical integrator. The VFODI response presented in Figure 5(b) is similar to the response of the ideal summator with lag.

(b) Now we consider decreasing order function given by

$$
\nu_{2}(k)=1+e^{-0.01 k} \text {. }
$$

The graph of the order function (36) is given in Figure 6(a). Values of order function begin at 2 and monotonically tend to 1 . This means that summation force successively declines to the classical summator. The response is shown in Figure 6(b).

Example 13. The VFOIE possesses also the property related to the classical integrator. For zero input signal and nonzero initial conditions, it preserves a nonzero output. In this example, we split our consideration to two different initial conditions:

$$
\begin{aligned}
& \mathbf{y}_{-1}^{1}=\left[\begin{array}{l}
1 \\
0 \\
0 \\
0 \\
\vdots
\end{array}\right], \\
& \mathbf{y}_{-1}^{2}=\left[\begin{array}{l}
0 \\
0 \\
1 \\
0 \\
\vdots
\end{array}\right] .
\end{aligned}
$$




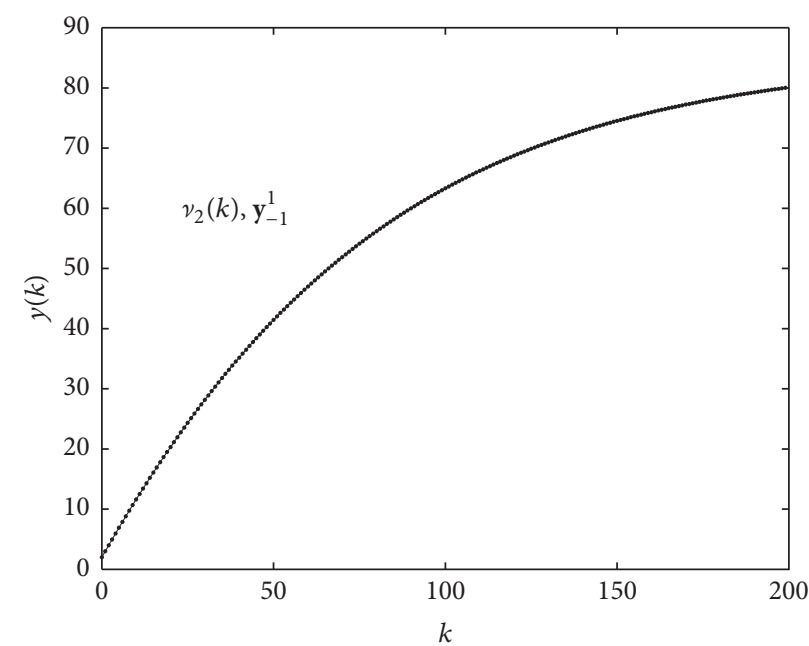

(a) Plot of response to $\mathbf{y}_{-1}^{1}$

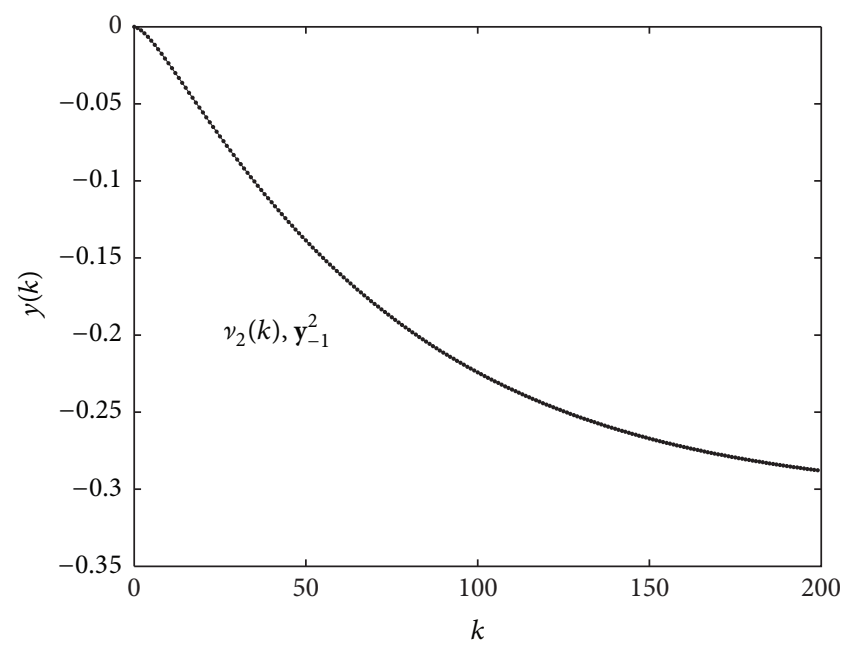

(b) Plot of response to $y_{-1}^{2}$

FIGURE 8: The VFOI homogenous responses to $\mathbf{y}_{-1}^{1}$ and $\mathbf{y}_{-1}^{2}$ with $b_{0}=0.001, k_{0}=0$ in formula (34) and the order $v_{2}(k)=1+e^{-0.01 k}$.

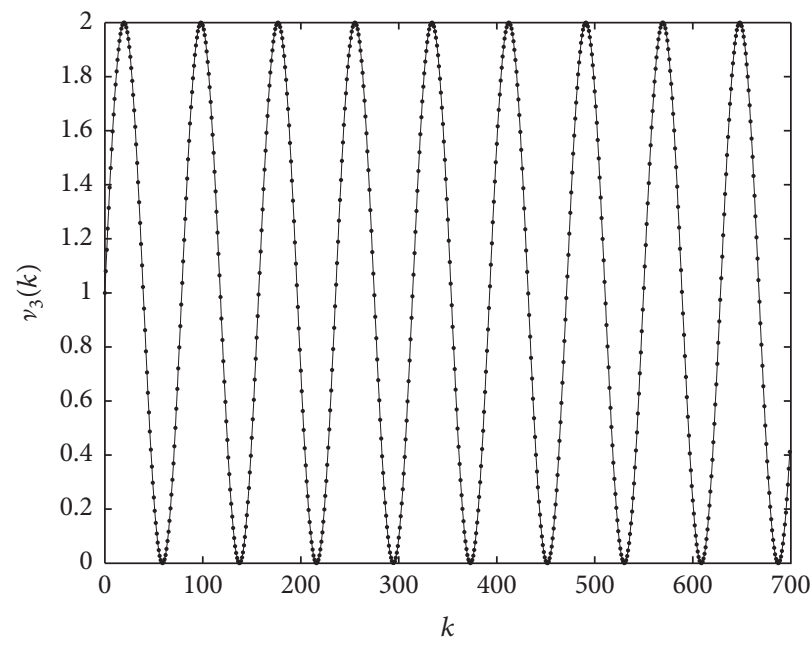

(a) Sinusoidal order function

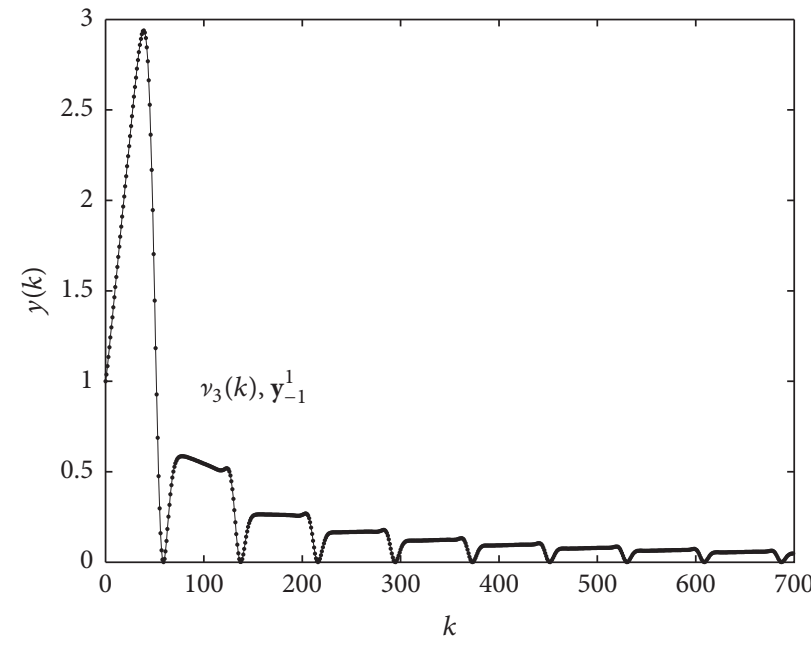

(b) The VFOI homogenous response to $\mathbf{y}_{-1}^{1}$

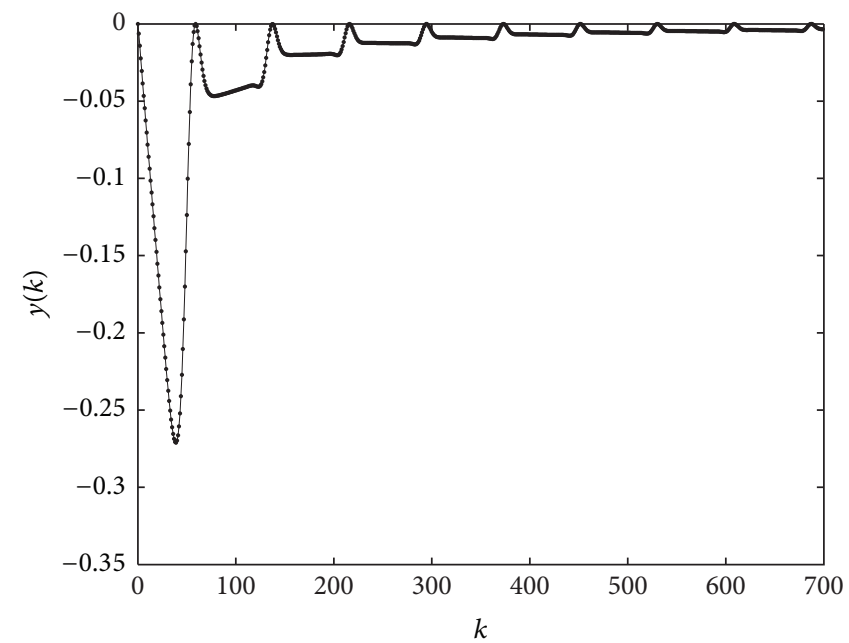

(c) The VFOI homogenous response to $\mathbf{y}_{-1}^{2}$

FIGURE 9: Plots of sinusoidal order function $v_{3}(k)=1+\sin (\pi k / 40)$ and the VFOI homogenous response to $\mathbf{y}_{-1}^{1}$ and $\mathbf{y}_{-1}^{2}$ represented by formula (34) with $b_{0}=0.001, k_{0}=0$. 
We change in the item order functions, considering increasing and decreasing order functions.

(c) For the order function $\nu_{1}(k)=1-e^{-0.01 k}$, we obtain the VFOIE homogenous responses presented in Figures 7 (a) and $7(\mathrm{~b})$, respectively. Indeed responses differ a little from each other. The second response reaches slightly lower steady state. This statement confirms that the oblivion function decreases due to the initial condition placed in the past. For the order function $v_{2}(k)=1+e^{-0.01 k}$, we obtain the VFOIE homogenous responses presented in Figures 8(a) and 8 (b), respectively.

(d) In this part, we examine the homogenous response to $\mathbf{y}_{-1}^{1}$ with a periodic order function given by $v_{3}(k)=$ $1+\sin (\pi k / 40)$. The plots of order functions and the simulated solutions are presented in Figures 9(a), 9(b), and 9(c), respectively. Values of both responses are tending to zero.

(ii) Let us take $v(k) \equiv 0$. Then, (25) takes the form

$$
Y(k)=b_{0}\left({ }_{k_{0}} \mathbf{A}_{k}^{[-\mu(k)]}\right) \mathbf{u}(k)
$$

and the VFODI response, with initial condition $Y\left(k_{0}-\right.$ 1) $=\mathbf{y}_{k_{0}-1}$, is described by the formula proved in Proposition 9:

$$
\mathbf{y}(k)=b_{0 k_{0}} \mathbf{A}_{k}^{[-\mu(k)]} \mathbf{u}(k)-{ }_{-\infty} \mathbf{I}_{k_{0}-1}^{[\nu(k)]} \mathbf{y}_{k_{0}-1} .
$$

\section{Final Conclusions}

The form of the variable-, fractional-order difference integrator (VFODI) is characterised by the two independent fractional-order functions. Both order functions are assumed to be nonnegative. There is no restriction concerning their equality. There is an immense choice of the fractional-order selection. One of the promising choices appears to be a relation of the order function with the input and output signals $v[|u(k)|]$ and $\mu[|u(k)|]$ or $v[|y(k)|]$ and $\mu[|y(k)|]$. In the closed-loop systems with VFO PID controller, the order functions can be related to the closed-loop error signal.

\section{Conflicts of Interest}

The authors declare that they have no conflicts of interest.

\section{Acknowledgments}

This research was partially supported by the Bialystok University of Technology Grant S/WI/1/2016 (Dorota Mozyrska) and the Lodz University of Technology Grant 501\12-241-5437 (Piotr Ostalczyk) and funded from the sources for research by Ministry of Science and Higher Education.

\section{References}

[1] T. Kaczorek, Linear Control Systems: Analysis of Multivariable Systems, John Wiley \& Sons, Inc, New York, NY, USA, 1992.
[2] T. Kailath, Linear Systems, Prentice-Hall, Englewood Cliffs, NJ, USA, 1980

[3] R. Abu-Saris and Q. Al-Mdallal, "On the asymptotic stability of linear system of fractional-order difference equations," Fractional Calculus and Applied Analysis. An International Journal for Theory and Applications, vol. 16, no. 3, pp. 613-629, 2013.

[4] D. Baleanu, K. Diethelm, E. Scalas, and J. J. Trujillo, Fractional Calculus Models and Numerical Methods, vol. 3 of Series on Complexity, Nonlinearity and Chaos, World Scientific, Singapore, 2012.

[5] D. Baleanu, J. A. T. Machado, and A. C. J. Luo, Fractional Dynamics and Control, Springer-Verlag, New York, NY, USA, 2012.

[6] R. Caponetto, G. Dongola, G. Fortuna, and I. Petras, Fractional Order Systems: Modeling and Control Applications, World Scientific, Singapore, 2010.

[7] S. Das, Functional Fractional Calculus for System Identification and Controls, Springer-Verlag, Berlin-Heidelberg, Germany, 2009.

[8] R. A. Ferreira and D. F. Torres, "Fractional $h$-difference equations arising from the calculus of variations," Applicable Analysis and Discrete Mathematics, vol. 5, no. 1, pp. 110-121, 2011.

[9] D. Mozyrska and M. g. Wyrwas, “The Z-transform method and delta type fractional difference operators," Discrete Dynamics in Nature and Society, vol. 2015, Article ID 852734, 12 pages, 2015.

[10] I. Podlubny, Fractional Differential Equations, vol. 198, Academic Press, San Diego, Calif, USA, 1999.

[11] A. Halanay and J. Samuel, Differential Equations, Discrete Systems and Control, Kluwer Academic Publishers, Dordrecht, Netherlands, 1997.

[12] E. C. Ifeachor and B. W. Jervis, Digital Signal Processing, Addison-Wesley, Edinburgh Gate, UK, 1993.

[13] F. M. Atici and P. W. Eloe, "A transform method in discrete fractional calculus," International Journal of Difference Equations, vol. 2, no. 2, pp. 165-176, 2007.

[14] M. A. Al-Alaoui, "Novel digital integrator and differentiator," Electronics Letters, vol. 29, no. 4, pp. 376-378, 1993.

[15] N. R. O. Bastos, R. A. C. Ferreira, and D. F. M. Torres, "Discretetime fractional variational problems," Signal Processing, vol. 91, no. 3, pp. 513-524, 2011.

[16] D. Mozyrska, "Multiparameter fractional difference linear control systems," Discrete Dynamics in Nature and Society, vol. 2014, Article ID 183782, 8 pages, 2014.

[17] M. D. Ortigueira, F. J. V. Coito, and J. J. Trujillo, "Discretetime differential systems," Signal Processing, vol. 107, pp. 198-217, 2015.

[18] P. Ostalczyk, Discrete Fractional Calculus: Applications in Control and Image Processing, vol. 14 of Series in Computer Vision, World Scientific Publishing Co Pte Ltd, Singapore, 2016.

[19] R. Stanisławski and K. J. Latawiec, "Stability analysis for discrete-time fractional-order LTI state-space systems. Part I: New necessary and sufficient conditions for the asymptotic stability, Bulletin of the Polish Academy of Sciences: Technical Sciences, vol. 61, no. 2, pp. 353-361, 2013.

[20] R. Stanisławski and K. J. Latawiec, "Stability analysis for discrete-time fractional-order LTI state-space systems. Part II: new stability criterion for FD-based systems," Bulletin of the Polish Academy of Sciences: Technical Sciences, vol. 61, no. 2, pp. 363-370, 2013.

[21] J. Cervera, A. Baños, C. A. Monje, and B. M. Vinagre, “Tuning of fractional PID controllers by using QFT," in Proceeding of the 
32nd Annual Conference on IEEE Industrial Electronics (IECON '06), pp. 5402-5407, Paris, France, November 2006.

[22] P. Ostalczyk and D. Mozyrska, "The second form of the variable-, fractional-order discrete-time integrator," in Proceedings of the 21st International Conference on Methods and Models in Automation and Robotics, MMAR 2016, pp. 859-864, pol, September 2016.

[23] D. Sierociuk, W. Malesza, and M. Macias, "On a new symmetric fractional variable order derivative," in Theoretical developments and applications of non-integer order systems, S. Domek and P. Dworak, Eds., vol. 357, pp. 29-39, Springer, Cham, Heidelberg, Germany, 2016.

[24] D. Mozyrska and P. Ostalczyk, "Variable-fractional-order Grünwald-Letnikov backward difference selected properties," in Proceedings of the 39th International Conference on Telecommunications and Signal Processing, TSP 2016, June 2016.

[25] A. A. Kilbas, H. M. Srivastava, and J. J. Trujillo, Theory and Applications of Fractional Differential Equations, Elsevier, Amsterdam, Netherlands, 2006.

[26] A. Oustaloup, La dérivation non entière: théorie, synthèse et applications, Hermes, Paris, France, 1995. 


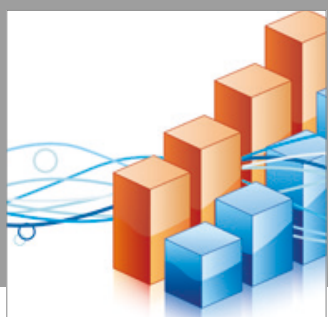

Advances in

Operations Research

vatersals

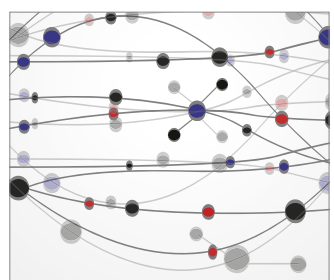

\section{The Scientific} World Journal
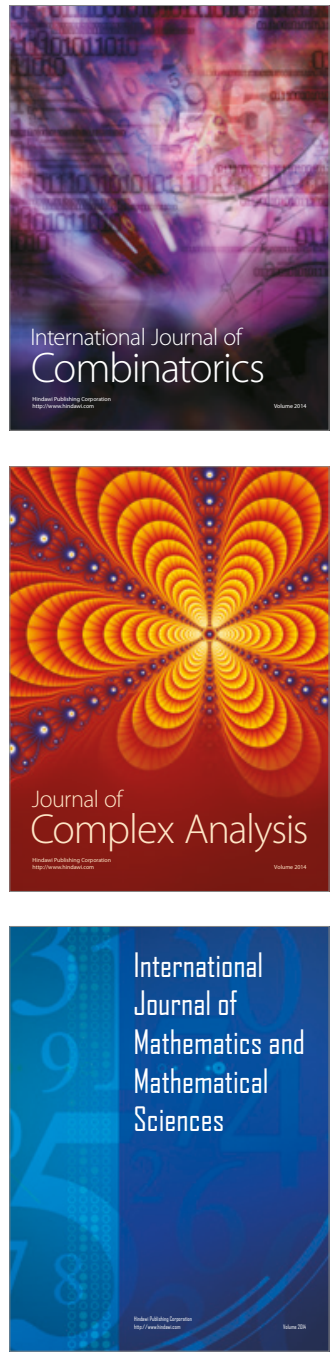
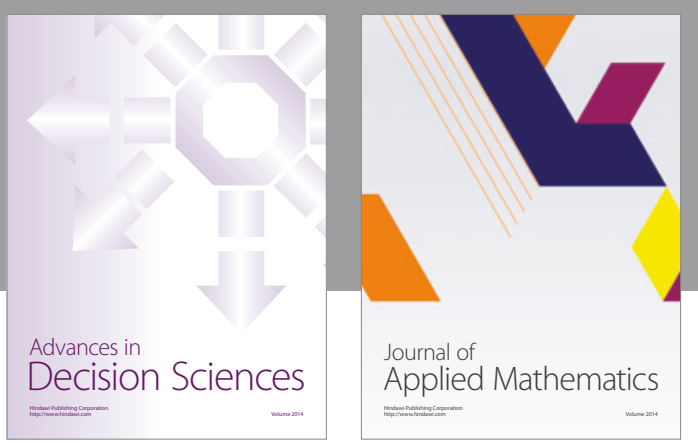

Algebra

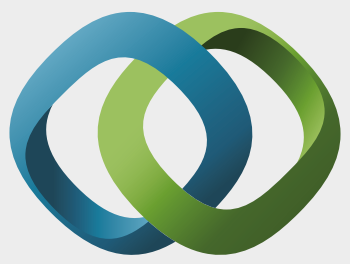

\section{Hindawi}

Submit your manuscripts at

https://www.hindawi.com
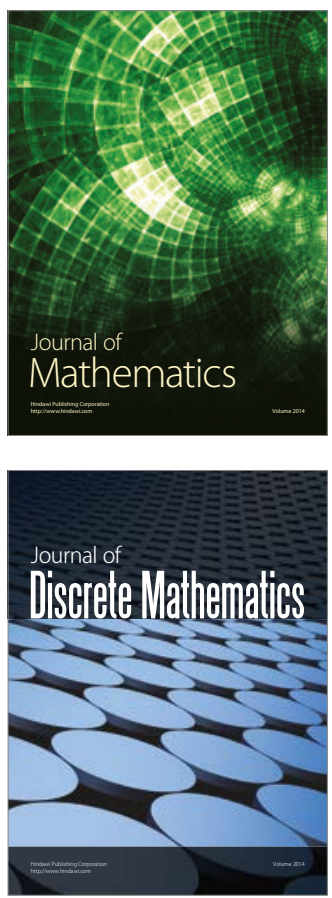

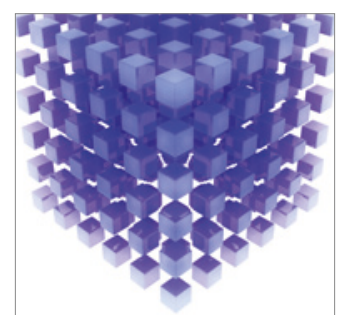

Mathematical Problems in Engineering
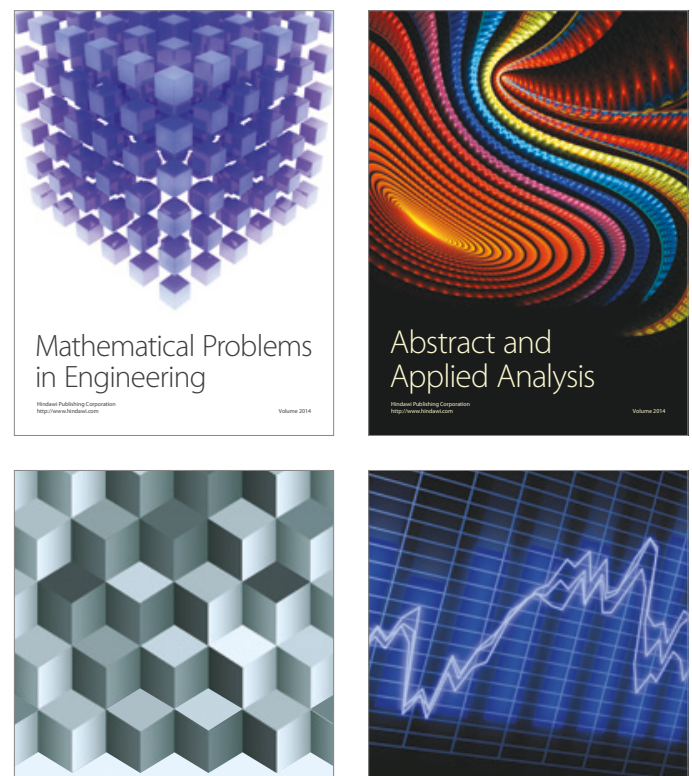

Journal of

Function Spaces

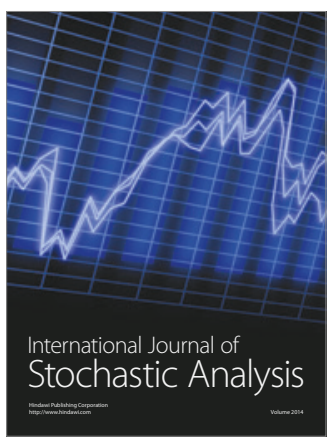

Probability and Statistics
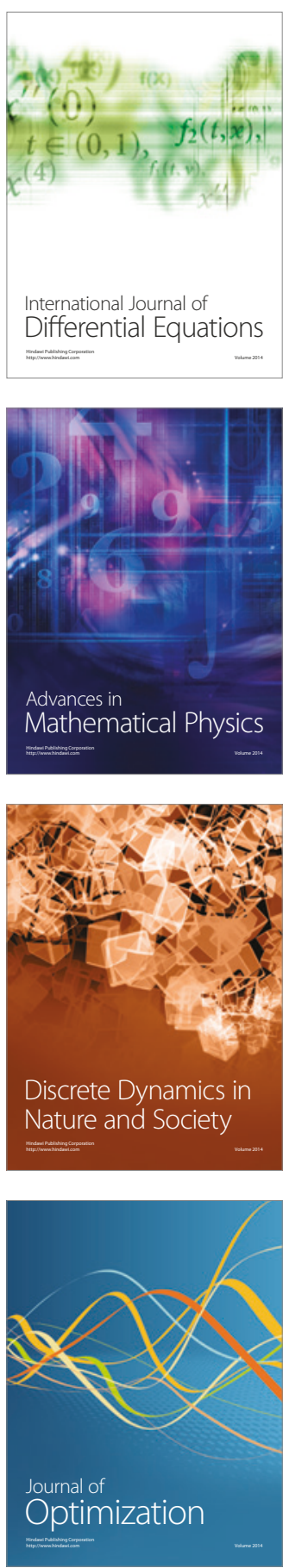